Dermatologische Zeitschrift. 1902;9:I-VI

\title{
Contents, Vol. 9, 1902
}

\section{HEFT i.}

Sei $\mathrm{t} \ll$ I. Betrachtungen iiber das Jodipin. (Erster Teil.)

Von Dr. Ernst J. Feibes in Aaclieu 1

II. Die Verwendung der Brooke'schen Pasta bei in-fektiösen und entzündlichen Hautaffektionen. Von Dr. Dreyer in Kölu 19

III. Die Dermatitis exfoliativa Wilson und das Erythema scarlatiniforme recídivans. Von Dr.

Friedrich Luithlen in Wien 89

IV. Die miki'oskopische Technik im Dienste der Dermatologie. Ein Rückblick auf die Jahre 1895 - 1900. (Erster Teil.) Von Dr. R. Leder-mann in Berlin und Dr. ${ }^{\wedge}$ S. Blanck in Potsdam . 4;3 Berliner dermatologische Gesellsc liaf't.

Sitzung void 5. November $1901 \quad 60$

Niederländische dermatologische Gesellschaft.

Bericht iiber das Jahr 1899 -1900 . . . 73 Vereinigung í'ranzösischer Urologen.

Fünfte Versammhmg, am 24.-26. Oktober 190176

Besprechungen. . . 79

Periodische Litteratur 90

Buchanzeigen 118

T agesnachri ch ten and Notizen

HEFT 2.

V. Strichförmige Havtausschläge am Bein. Von

Dr. L. Fischel und Dr. F. Pinkus in Berlin . . 123

VI. Beiträge zur Casuistik seltener gon orrhoischer

Erkrankungen. Von Privatdozent Dr. Jtilius Heller

in Berlin 130

।: II. U eber das V orlí o mm en des B a c t erium co 1 i com-mune bei postgonorrhoischer Urethritis. Von Dr. Reinhold Ledermann in Berlin .... 139 VIII. Praktische Methode der Prophvlaxe des Trippers.

Von Professor Claudio Fermi in Jassari 144

IX. Ergebuisse der phototherapeutischen Behandlung bei tuberculösem Lupus des Gesichts. Von Dr. Leredde und Dr. Pan trier in Paris . . . 153

$-\mathrm{IV}$ -

X. Bill Fall diffuser chronischer Talgdrüseühyper-trophie. Von Dr. Antonino Marullo in Berlin. (Hierzu Tafel I) . . , . . 166

XI. Das Atoxyl (Metaarsensäureanilid), ein neues Arsenpräparat, i $\prod u l$ dessen detmatotherapeutische Verwendong. Yro $\_$Dr. Wather Schild in Berlin 172

XII. Betrachtungeu fiber das Jodipin. — (Fortsetzung.)

Von Dr. Ernst J. Feibes in Aachen 182 
Ill. Die mikroskopische Technik im Di e nste der Dermatolo gie. Ein Rǘckblick auf die Jahre 1895-1900. (Fortsetzung) Von Dr. R. Leder-mann in Berlin und Dr. ¿*. Bíanck in Potsdam . 199 Berliner dermatologische Gesellschaft.

Sitzung vom 3. Dezember 1901216

Dänische dermatologische Gesellschaft.

Sitzung vom 2. Oktober $1901 \ldots . . .232$

Periodische Litteratur 235

B es prechungen 286

Buchanzeige 289

Tagesnachrichten und Notizen 290

Nachruf an Moritz Kaposi 291

HEFT 3.

'IV. Erythema scarlatiniforme desquamativum reci-

divans. Von Dr. Julius Kramsztyk in $\Lambda$ Varschau 293 XV. Die Parrot'schen Pseudoparalysen bei ange-borener Syphylis. Von Prof. Dr. Franz Schever

in Prag 305

“"M. Betrachtungen über das Jodipin. (Schluss.) \"'on

Dr. Ernst J. Peibes in Aachen ..... . . 329

VII. Die mikroskopische Technik im Dienste der Dermatolo gie. Ein Rückblick auf die Jahre 189

5-1900. (Fortsetzung.) Von Dr. R. Ledßr-mann in Berlin und Dr. S. Bíanck in Potsdam . 347 .

Untersuchungen über die Toxicität des Blut-serums bei fvischer Pellagra und über die

antitoxische Kraft des Blutserums geheilter Pellagrakran ker. Von Dr. G. Antonini und Dr.

A. Mariani 458

Berliner dermatologische Gesellschaft.

Sitzung vom 7. Januar 1902368

Niederländische dermatologische Gesellschaft.

Bericht über das Jahr 1900-1901 .... 380

Periodische Litteratur 389

Besprechungen 443

Buchanzeigen 449

Personalien und Tagesnachrichten ..... 463

Nachruf an Hans von Hebra 464

$-\mathrm{V}-$

HEFT 4.

XIX. Weber einen bisher unbekannten Nebenap pavat am Haarsystem des Menschen:

Haarscheiben.

Von Dr. Felix Pinlms in Berlin 465

XX. Qnecksilbervergiftung mit tödtlichem Ausgange

nacli Einspritzungen von Hydrargyrum sali

cylic um. Von Dr. R. Neubeck in Berlin . . 470

XXI. Eine neue Behandlungsmethode der Gonorrhoe.

Von Dr. Albert MarcMS in Müncben508

XXII. Гeber praepiitiale Schleimhautgänge mit Little-schen Schleim clrüsen und deren gonorrhoiscbe Erkrankung. Von Dr. Johannes Fick in Wien.

(Hierzu Tafel ü and III) 516 
XXIIl. Die mikroskopisclie Technik im Dienste der Dermatologie. Ein Riickblick auf die Jahre 1895 bis 1900. (Fortsetzung.) Von Dr. R. Ledermann in Berlin und Dr. »S. Blanck in Potsdam . . . 531 Berliner dermatologische Gesellschaft.

Sitzung voin 2. Februar 1902.541

Dänische dermatologische Gesellschaft.

Sitzung torn 6. November $1901 \quad 54 \mathrm{~s}$

Sitzung voin 4: Dezember 1901552

Periodische Litteratur . 555

Besprechungen 585

Buchanzeigen 594

Tagesnaehrichten und Notizen $\quad 60 \prod$

Eine neue japanische Zeitschrift 601

Personalien 602

HEFT 5.

XXIV. Ueber Hau tafíektionen nacli dem Gebrauche

von Aureol. \Гon Prof. Dr. M. Wolters in Rostock 608 XXV. Ein Fall von Arse n intox icíition.

Von Dr. O. Rosen-

thal in Berlin 609

XXVI. Die Zahndifformitäten in der Diagnose der here-ditären Syphilis. Von Dr. Franz Poor m Budapest. (Aus dem dermatologischen Institute der königl. ungar.

Ùniversität zu Budapest $\left.{ }^{\wedge}\right) \quad 616$

XXVII. Erfahrungen über die Behandlung und $1 \cdot$ ieilung

der L·epra im Riga'schen Städtischen Lepro-

sorium. Von Dr. Matthias Hirschberg in Riga . 626

XXVIII. Ueber einen Fall von Granuloma tricliophyticum

Majocchi. Von Prof. P. Colombini in Sassari, deutsch

von M. Dreysel in Leipzig. (Aus der Ciinica Dermosi-

tilopatica della R. üuiversitá di Sassari diretta dal Prof.

P. Colombini) 641

XXIX. Die mikroskopisclie Technik im Dienste der Dermatologie Ein Rückblick auf die Jahre 1895

vi -

bis 1900. (Schluss.) Von Dr. R. Ledermann in

Berlin und Dr. ^, Blanck in Potsdam .... $6 \Gamma>1$

XXX. DieBelehrung desPubliku nis über die v en e rise lien

Krankheiten. Von Dr. O. Lassar in Berlin . . 664

Berliner dermatologisclie Gesellschaft.

Sitzung vom 4. März $19.02 \quad 67 \mathrm{a}$

Sitzung vom 6. Mai $1902 \quad 684$

II. Internationale Konferenz zur Propbylaxe der Syphilis und der venerischen Krankheiten zn

Brüssel vom 1.-6. September 1902 .... 693

Periodisclie Litteratur $\quad 728$

Besprechungen 76ÍJ

Buchanzeigen $\quad 772$,

Tagesnachrichteu 77,'!

Nachruf an Rudolf Virehow 77:! 


\section{HEFT 6.}

XXXI. üeber Wirkuugen constanter Wärme auf die Haut und andere Or gaii e mit Demonstration des Hydrother moregulators und verschiedener Thermokörper. Yon Priv.-Doc. Dr. Karl Ullmann in Baden b. $\Lambda$ Vien $77 \Gamma>$

XXX1l. Ein schweres, spat eingetvetenes. bullöses Queck-silberexanthem nach 12 Einreibungen mit grauer Salbe. $\Lambda^{5} / 8 n$ Dr. P. Thimm in Leipzig . 782

XXXIII. $\quad$ L·ichttherapeutische Spezialitäten und neue

Lichtgeneratoren. Von Dr. Hermann Strebeí

in Miinchen $79 . ' i$

XXXIV. Eigenthümliche leichte Auszieh barkeit der

Papillenh aare und ihrer Wurzelseheiden. Von

61. Giovanniní in Turin 809

Die Radiotherapie an dem II. Internationalen Congress für medicinische Elektrologie und

Radiologie. Bern, I. - 6. September 1902 . . 812

Berliner dermatologisclie Gesellschaft.

Sitzung vom 3. Juni $1902 \quad 817$

Verhandlnngen der Abtheilung für Dermatologie

und Syphilis der 74. Versammlung deutscher

Naturforscher und Aerzte in Karlst $>$ ad vom

21.-27. September $1902 \quad 831$

Bericht über die constituirende Versammlung der Deutschen Gesellschaft zur Bekämpfung der Geschlechtskrankheiten am 19. Oktober 1902875

Besprechungen $\quad .888$

Buchanzeigen 901

Tagesuachrichten 908 